\title{
Binary Population and Spectral Synthesis
}

\author{
J. J. Eldridge ${ }^{1} \dagger$, E. R. Stanway ${ }^{2}$, L. Xiao ${ }^{1}$, L. A. S. McClelland ${ }^{1}$, \\ J. C. Bray ${ }^{1}$, G. Taylor ${ }^{1}$ and M. Ng$^{1}$ \\ ${ }^{1}$ Department of Physics, University of Auckland, Private Bag 92019, Auckland, New Zealand \\ ${ }^{2}$ Department of Physics, University of Warwick, Gibbet Hill Road, Coventry, CV4 7AL, UK
}

\begin{abstract}
We have recently released version 2.0 of the Binary Population and Spectral Synthesis (BPASS) population synthesis code. This is designed to construct the spectra and related properties of stellar populations built from $\sim 200,000$ detailed, individual stellar models of known age and metallicity. The output products enable a broad range of theoretical predictions for individual stars, binaries, resolved and unresolved stellar populations, supernovae and their progenitors, and compact remnant mergers. Here we summarise key applications that demonstrate that binary populations typically reproduce observations better than single star models.
\end{abstract}

Keywords. methods: numerical, stars: evolution, stars: statistics, galaxies: stellar content

BPASS stellar models were first introduced in 2009 and publically released in 2012. Our v2.0 release in mid-2015 was the most complete and widely-usable to date. This has been reflected in refereed publications using the models in a variety of contexts, including:

\section{Stellar and Transient:}

(i) Rates and lightcurves of electron-capture supernovae (Moriya \& Eldridge 2016)

(ii) The progenitors of Type Ib SNe (Eldridge \& Maund 2016)

(iii) Rates of mergers of compact stellar remnants and their role as gravitational wave transient progenitors (Eldridge \& Stanway 2016)

(iv) Evolutionary channels for WR stars in the SMC (Shenar et al. 2016)

\section{Extragalactic and Cosmological:}

(i) Ionizing photon production at low metallicities and implications for the epoch of reionization (e.g. Stanway, Eldridge \& Becker 2016)

(ii) Fitting the integrated light and photometry of young stellar clusters in nearby galaxies (e.g. Wofford et al. 2016)

(iii) Stellar populations in galaxies in the distant Universe (e.g. Strom et al. 2017)

Other results are discussed in the articles by the co-authors elsewhere this proceedings. We are currently preparing a methodology and verification paper for BPASS (Eldridge et al., in prep), which will be released together with v2.1 of the models. BPASS and its outputs can be found at http://bpass.auckland.ac.nz.

\section{References}

Eldridge J. J., Stanway E. R., 2016, MNRAS, 462, 3302

Eldridge J. J., Maund J. R., 2016, MNRAS, 461, L117

Moriya T. J., Eldridge J. J., 2016, MNRAS, 461, 2155

Shenar T., et al., 2016, A\&A, 591, A22

Stanway, E. R., Eldridge, J. J., \& Becker, G. D. 2016, MNRAS, 456, 485

Strom A. L., et al., ApJ, 836, 164

Wofford A., et al., 2016, MNRAS, 457, 4296

$\dagger$ email: j.eldridge@auckland.ac.nz 